University of Nebraska - Lincoln

DigitalCommons@University of Nebraska - Lincoln

Food and Drug Administration Papers

U.S. Department of Health and Human Services

2012

\title{
Characterization of Pasteurella multocida strains isolated from geese
}

\author{
Zsuzsanna Varga \\ Hungarian Academy of Sciences,, vargazs@vmri.hu \\ Dmitriy V. Volokhov \\ Food and Drug Administration \\ Laszlo Stipkovits \\ RT-Europe Research Center \\ Akos Thuma \\ Veterinary Diagnostic Directorate \\ Boglarka Sellyei \\ Hungarian Academy of Sciences,
}

See next page for additional authors

Follow this and additional works at: https://digitalcommons.unl.edu/usfda

Part of the Dietetics and Clinical Nutrition Commons, Health and Medical Administration Commons, Health Services Administration Commons, Pharmaceutical Preparations Commons, and the Pharmacy Administration, Policy and Regulation Commons

Varga, Zsuzsanna; Volokhov, Dmitriy V.; Stipkovits, Laszlo; Thuma, Akos; Sellyei, Boglarka; and Magyar, Tibor, "Characterization of Pasteurella multocida strains isolated from geese" (2012). Food and Drug Administration Papers. 53.

https://digitalcommons.unl.edu/usfda/53

This Article is brought to you for free and open access by the U.S. Department of Health and Human Services at DigitalCommons@University of Nebraska - Lincoln. It has been accepted for inclusion in Food and Drug Administration Papers by an authorized administrator of DigitalCommons@University of Nebraska - Lincoln. 


\section{Authors}

Zsuzsanna Varga, Dmitriy V. Volokhov, Laszlo Stipkovits, Akos Thuma, Boglarka Sellyei, and Tibor Magyar 


\title{
Characterization of Pasteurella multocida strains isolated from geese
}

\author{
Zsuzsanna Varga a,*, Dmitriy V. Volokhov ${ }^{\mathrm{b}}$, László Stipkovits ${ }^{\mathrm{c}}$, Ákos Thuma ${ }^{\mathrm{d}}$, \\ Boglárka Sellyei ${ }^{\mathrm{a}}$, Tibor Magyar ${ }^{\mathrm{a}}$ \\ a Institute for Veterinary Medical Research, Centre for Agricultural Research, Hungarian Academy of Sciences, P.O. Box 18, H-1581 Budapest, Hungary \\ ${ }^{\mathrm{b}}$ Center for Biologics Evaluation and Research, Food and Drug Administration, 1401 Rockville Pike, HFM-470, Rockville, MD 20852, USA \\ ${ }^{\mathrm{c}}$ RT-Europe Research Center 9200, Var 2, Mosonmagyarovar, Hungary \\ ${ }^{\mathrm{d}}$ National Food Chain Safety Office, Veterinary Diagnostic Directorate, Tábornok u. 2, H-1143 Budapest, Hungary
}

\section{A R T I C L E I N F O}

\section{Article history:}

Received 19 April 2011

Received in revised form 20 December 2012

Accepted 22 December 2012

\section{Keywords:}

Pasteurella multocida

Goose

Fowl cholera

Pasteurellosis

\begin{abstract}
A B S T R A C T
Phenotypic and genotypic diversity of forty-two Pasteurella multocida isolates from geese were characterized by analysis of their capsular type, Heddleston serotype, biotype, fimbrial gene allele type, comparative outer membrane protein (OMP) electrophoresis patterns, and were analyzed using PCR for the presence of virulence-associated genes (toxA, $t b p A$, pfh $A$, $h g b A, h g b B, n a n H, n a n B$, fimA, $h s f-1$, and $p m H A S$ ). A sequence comparison of the thdF and $r p o B$ housekeeping genes of twenty representative $P$. multocida strains from three different OMP groups demonstrated that seventeen strains were closely related phylogenetically to previously published strains of $P$. multocida subsp. multocida and $P$. multocida subsp. gallicida, and only three strains from geese were grouped with previously published strains of $P$. multocida subsp. septica. This study is the first report regarding the characterization of phenotypic and genotypic features of different $P$. multocida field strains isolated from geese with the different clinical representation of pasteurellosis and provide our overview on the usefulness of these in vitro tests for primary characterization of $P$. multocida strains for the epidemiological studies of fowl cholera.
\end{abstract}

(c) 2013 Elsevier B.V. All rights reserved.

\section{Introduction}

Pasteurella multocida is the causative agent of fowl cholera (FC), a contagious disease that affects over 100 avian species worldwide and leads to significant economic losses in domestic and commercial poultry (Christensen and Bisgaard, 2000; Harper et al., 2006). In the European Union, FC has been eliminated from closed-cycle commercial large scale chicken and turkey flocks; however, disease can still be found in water fowl kept in semi-closed husbandry systems where free access to the outside environment and water is allowed.

\footnotetext{
* Corresponding author at: Institute for Veterinary Medical Research, Hungarian Academy of Sciences, Hungária krt. 21, 1143 Budapest, Hungary. Tel.: +361467 4060; fax: +36 14674076 .

E-mail addresses: vargazs@vmri.hu, varga.zsuzsanna.iren@gmail.com (Z. Varga).
}

$P$. multocida has been classified by serological methods into five capsular serogroups designated A, B, D, E and F, and 16 serotypes can be distinguished by analysis of heat stable precipitin antigens (Christensen and Bisgaard, 2000; Heddleston et al., 1972). Three subspecies of $P$. multocida have been established (subsp. multocida, subsp. septica and subsp. gallicida) based on results of total genomic DNA hybridization, carbohydrate fermentation, enzymatic activities, and phylogenetic gene sequence comparison studies (Davies, 2004; Hunt Gerardo et al., 2001; Kuhnert and Korczak, 2006; Mutters et al., 1985). Based on differences in fermentation activities, $P$. multocida isolates have been classified into 13 different biotypes (Blackall et al., 1997; Fegan et al., 1995). In addition, more than 60 different outer membrane proteins (OMP), with presumed roles in pathogenesis and immunity, have been identified in $P$. multocida strains isolated from a wide range of animal species (Hatfaludi et al., 2010). Fimbriae of $P$. multocida 
encoded by the $p t f A$ gene may play an important role in adhesion of the bacterium to the host epithelial cells, and the ptfA gene polymorphism can be a useful tool for epidemiological studies (Sellyei et al., 2010; Tang et al., 2009).

Domestic and wild geese are frequently affected by FC (avian pasteurellosis) (Blanchong et al., 2006; Samuel et al., 2005). The strains of $P$. multocida detected in geese exhibit substantial differences in their virulence and therefore in their ability to cause peracute, acute or chronic forms of FC. At this time, no serological, biochemical or genetic markers are known that permit the differentiation of highly virulent strains from less virulent strains of $P$. multocida isolated from poultry. Experimental animal infection (Wilkie et al., 2000 ) is still a "gold standard" method for assessing the potential virulence of $P$. multocida isolates. In addition, the phenotypic and genotypic characteristics of $P$. multocida strains and its subspecies associated with the different clinical representation of pasteurellosis in geese are still unknown.

Thus, the main goal of this study was to analyze the phenotypic and genotypic characteristics of $P$. multocida field strains isolated from geese afflicted with different forms of pasteurellosis. This study summarizes the characterization of $P$. multocida strains isolated from geese in Hungary and provides our overview on the diversity of $P$. multocida field strains based on in vitro testing results that may reflect to the potential virulence of $P$. multocida isolates.

\section{Materials and methods}

\subsection{P. multocida strains studied and cultural conditions}

Phenotypic and genotypic characteristics of $42 P$. multocida isolates from geese were studied. Of these 42 P. multocida strains, 34 strains were isolated from geese with confirmed diagnoses of FC at the Central Veterinary Diagnostic Institute of Hungary in 2005-2009. To choose strains representative of a diverse epidemiological setting, strains were selected taking into account the locations and time of their isolation in Hungary as well as the available data on disease cases. Based on the disease profile associated with these strains, these FC isolates were separated into three groups: (i) strains from cases of peracute fowl cholera ( $\mathrm{pFC}$ ) in geese, which were characterized by fulminating acute fatal septicaemia with high mortality in the geese flocks, (ii) strains from cases of typical acute FC ( $\mathrm{FFC}$ ) where the infections developed more slowly and with less fatalities, and (iii) strains from chronic atypical (so-called "pasteurellosis") cases which were characterized by local, non-systemic inflammations in the respiratory tract, joints, peritoneal cavity or oviducts and caused low mortality in the geese flocks. Eight additional $P$. multocida strains, which were isolated from geese with not reported diagnoses (Table S1) in an earlier period (from 1990 to 2005), were used to compare characteristics among the strains of the focus group (2005-2009). All these P. multocida strains were directly isolated from liver, septic blood obtained from heart, or tissue lesions using sheep blood agar. In the cases diagnosed as pasteurellosis in geese (non-pFC/non-tFC cases), P. multocida strains were isolated from local nonsystemic infections; phallus inflammation (Strains P747, P898), ovarian inflammation (Strain P770/1), salpingitis (Strain P463), cloacitis (Strain P899) and a site of subcutaneous tissue inflammation (Strain P597). In addition, seven non-geese $P$. multocida strains isolated in Hungary from other species were studied as comparators to the strains isolated from geese (Table S1).

Fifty microliters of pure strains grown in brain-heart infusion broth were centrifuged at $16,000 \times \mathrm{g}$ for $10 \mathrm{~min}$. Total DNA from the cell pellet was extracted using a DNeasy blood and tissue kit (Qiagen, Chatsworth, CA) according to the manufacturer's protocol.

\subsection{Biochemical characterization, $P C R$ assays, and phylogenetic analysis of the strains}

Biochemical characterization, determination of subspecies of $P$. multocida, somatic serotype and biotype, $P$. multocida-specific PCR, capsule-specific multiplex PCR, and toxA gene-specific PCR were carried out as described elsewhere (Kuhnert and Korczak, 2006; Lichtensteiger et al., 1996; Mutters et al., 1985; Townsend et al., 1998; Varga et al., 2007). The typing of the ptfA gene for the strains was performed using PCR (Sellyei et al., 2010). The detection of the presence of virulence genes (toxA, tbpA, pfhA, $h g b A, h g b B$, $n a n H, n a n B$, fim $A, h s f-1$, and $p m H A S$ ) in all strains was carried out as described earlier (Ewers et al., 2006; Tang et al., 2009). In addition, amplification of the nanB and nan $H$ genes of $P$. multocida strains used in this study was carried out using the next in-house primers designed based on homologous nucleotide sequence regions in these full-length genes available at GenBank (CP003022, CP003313, CP001409, AF274868, and AF274869): nanB-Forw (TGGGGCGATATTGATACCGCAATCC), nanB-Rev (GGTCATTGACATCTGAAAGCGAGGC), nanH-Forw (TACGGTGGAAGACAGCAAGTGACC) and nanH-Rev (TCGGTCGAATACTCACACTGATATCCG). The sizes of $n a n B$ and $n a n H$-specific amplicons were 2888 bp and 1907 bp, respectively.

Briefly, the standard PCR mixture $(50 \mu \mathrm{l})$ contained 1.5 $\mathrm{U}$ of HotStarTaq-Plus DNA polymerase, $1 \times$ reaction buffer supplemented with $2.5 \mathrm{mM} \mathrm{MgCl2}$ (Qiagen, Chatsworth, CA), $500 \mathrm{nM}$ of each forward and reverse primer, a $200 \mu \mathrm{M}$ concentration of each deoxyribonucleoside triphosphate (dATP, dGTP, dCTP, and dTTP), and $7 \mu$ l of DNA template (ca. $0.1-0.2 \mu \mathrm{g}$ of total DNA). The PCR was performed using a GeneAmp PCR system model 9600 thermocycler (PE Applied Biosystems, Foster City, CA), with the following cycle conditions: initial activation at $95{ }^{\circ} \mathrm{C}$ for $5 \mathrm{~min}, 40$ cycles of $94^{\circ} \mathrm{C}$ for $1 \mathrm{~min}, 60^{\circ} \mathrm{C}$ for $1 \mathrm{~min}$, and $72{ }^{\circ} \mathrm{C}$ (extension) for $1.5 \mathrm{~min}$; and a final extension at $72{ }^{\circ} \mathrm{C}$ for $7 \mathrm{~min}$. The synthesis of PCR products with the expected molecular weights was confirmed by electrophoresis using a $1 \%$ SeaKem Gold TAE-agarose gel with ethidium bromide (Lonza, Allendale, NJ), followed by UV visualization.

Phylogenetic analysis of thdF and $r p o B$ gene sequences of selected strains was performed as described elsewhere (Kuhnert and Korczak, 2006; Petersen et al., 2001; Stahel 
et al., 2009). DNA sequences from this study were deposited in GenBank under accession numbers JF523357-JF523413, KC512818-KC512829.

\subsection{Outer membrane protein (OMP) profiling of the strains}

The OMP fractions were prepared as described previously (Davies et al., 1992, 2003). Sodium dodecyl sulphate polyacrylamide gel electrophoresis (SDS-PAGE) of the OMP fractions was carried out in $1.0 \mathrm{~mm}$ thick gel (4\% stacking and $15 \%$ separating) using Bio-Rad Protean II xi Cell equipment and run according to the Bio-Rad protocol. Before the loading step, the samples were mixed $(1 / 4 ; \mathrm{v} / \mathrm{v})$ with $2 \times$ sample buffer contained dithiothreitol and boiled for $5 \mathrm{~min}, 20 \mu \mathrm{g}$ protein of each sample was loaded into each well. Bio-Rad Precision Plus Protein Standard (10$250 \mathrm{kDa}$ ) was used as the molecular weight marker. The gel was run at constant current ( $30 \mathrm{~mA})$ as the samples moved through the stacking gel; the current was increase to $40 \mathrm{~mA}$ for the continuous run in the separating gel. The electrophoresed proteins were stained with Coomassie Brillant Blue R-250.

Gels were scanned and analyzed using Fingerprinting II software (Bio-Rad). Based on the comparative OMP profiling analysis of the $P$. multocida strains, the dendrogram representing the similarity among the protein patterns was constructed according to the Dice co-efficient.

\section{Results}

\subsection{Presumptive identification and general characteristics of the strains}

All the tested isolates were positive in the P. multocidaspecific PCR and negative in the P. multocida toxin (toxA) gene-specific PCR. Table S1 summarizes the clinical diagnoses in geese from which the strains were isolated and the observed characteristics of these strains, i.e. the capsule types, Heddleston somatic serotypes, biotypes, allele type of fimbrial ptfA gene, and the biochemical test results. Of the 42 P. multocida strains studied, 40 strains were found to express capsule type A and two strains (P898, P899) expressed capsule type F. Serotyping of the Heddleston somatic antigen of the strains revealed the presence of serotypes $1,3,3,4,(5)$ and 4,5 . In the biotype studies, biotype 3 and an arabinose fermenting unclassified biotype were found among the 21/42 and 15/42 of the geese strains, respectively; only a few strains expressed biotype 2 (3/42), 1 (1/42), and 7 (1/42). One P. multocida strain (P770/1, isolated from a goose with ovarian inflammation) fermented maltose and therefore this strain was considered an unclassified maltose-positive biotype. The maltose-positive strains of both $P$. multocida subsp. multocida and P. multocida subsp. septica have been isolated previously from different hosts including avian species (Fegan et al., 1995; Korbel et al., 1992; Petersen et al., 1998).

Thus, based on the results of the species-specific PCR assay and phenotypic testing, all these strains were recognized to be $P$. multocida species; however, their subspecies affiliation within the species was uncertain based on the biochemical data.

\subsection{Outer membrane protein (OMP) profiling}

The OMP-based profiling of $P$. multocida geese strains (Fig. 1) revealed a variety of protein bands ranging from approximately 20 to $150 \mathrm{kDa}$. Based on the similarity of the OMP protein patterns, these strains can be clustered in three main groups (Fig. 1). In addition, the third group can be subdivided in three subgroups (IIIa, IIIb, and IIIc).

Group I consists of only the capsule type F P. multocida strains isolated from two independent cases of pasteurellosis in two geese with local infections, i.e. phallus inflammation (P898) and cloacitis (P899). Group II contains only strains of $P$. multocida serotype A: 1 isolated from 1990 to 2008. This group represents all eight peracute FC strains studied with only a few strains isolated from geese with typical FC. All of the strains isolated from geese and assigned to group II expressed an unclassified biotype that was characterized by arabinose-positive and dulcitoland xylose-negative reactions and by the weak ability of these strains to ferment sorbitol (Table S1). In addition to these group II strains from geese, the seven $P$. multocida non-geese strains (Table S1) also expressed the same unclassified biotype characteristics and fell into the OMP group II (data not shown).

Subgroup IIIc consists of only one maltose-positive $P$. multocida strain (770/1) that exhibited a unique OMP electrophoretic pattern. Subgroup IIIb contains $P$. multocida strains that were found to express different biotypes and Heddleston somatic antigens; these strains were isolated predominantly from geese with "pasteurellosis" (i.e., non-systemic local) infections. Subgroup IIIa consists of strains isolated mainly from tFC cases and contained $P$. multocida strains with biotype 3 and one $P$. multocida strain (P964) that expressed biotype 2.

Thus, we can assume that the OMP pattern-based grouping of these 42 different strains may reflect to their phenotypic and/or genetic similarity, for example, all the strains classified as group II were arabinose-positive and included all the strains associated with peracute FC.

\subsection{Detection of virulence-associated genes}

The results of PCR analysis for the presence of virulence-associated genes ( $t b p A, p f h A, h g b A, h g b B, n a n H$, nanB, fim $A, h s f-1$, and pmHAS) are summarized in Table S2. All the tested isolates were positive for pmHAS (hyaluronan synthase), $h g b A$ (hemoglobin-binding protein A), $h g b B$ (hemoglobin-binding protein B), and fim $A$ genes and variable for the presence of $h s f-1, \operatorname{nanH}$ (sialidase $\mathrm{NanH}$ ), and $n a n B$ (sialidase NanB) genes. All the OMP group II strains, and maltose-positive strain P770/1 (OMP group IIIc) were found negative for both nanH and nanB sialidase genes using previously published primers (Tang et al., 2009). Two strains of the OMP group I were also found negative for nanB sialidase gene using the same primers. Because the real absence of $n a n H$ and $n a n B$ sialidase genes in all OMP group II and IIIc strains was uncertain for us the novel in-house primers for the amplification of nanH and nan $B$ were designed (see above) and used to clarify the situation. We found that all these strains were positive for nan $B$ sialidase gene with the novel primers. The nanB gene 


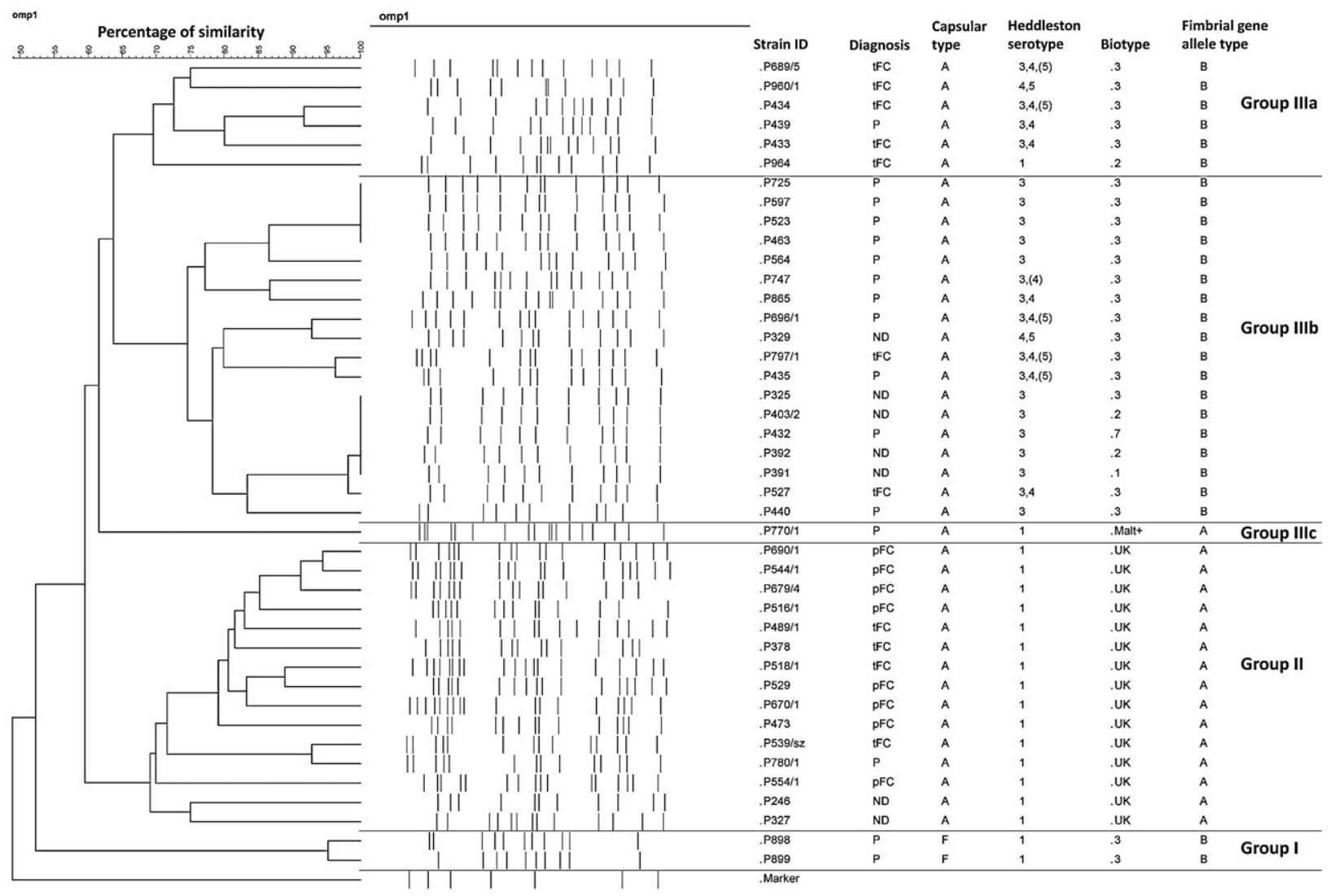

Fig. 1. The OMP pattern-based grouping of these studied 42 strains isolated from geese with different disease patterns of FC. Note: ND - no data; UK unknown; Malt+- unclassified maltose-positive biotype; P - "pasteurellosis" cases which were characterized by local non-systemic inflammations and low mortality in the geese flocks; pFC - peracute fowl cholera in geese; tFC - typical acute FC in geese.

amplicons sequenced from several strains (Fig. S1) of the OMP group II were phylogenetically closely related to the same gene in P. multocida 36950 (GenBank accession number CP003022). Unfortunately, due to the presence of mutations in the binding regions of the published primers within the gene (Tang et al., 2009), the amplification of nanB in these strains was failed using the primers. All our attempts to amplify nanH sialidase gene in all OMP group II and IIIc strains were unsuccessful with the novel in-house designed primers. However, due to substantial level of nucleotide sequence variability of $\mathrm{nanH}$ sialidase gene in $P$. multocida found in GenBank (data not shown), we cannot entirely consider that these strains lacking the gene. Thus, biochemical testing of sialidase activity in such strains seems to be most appropriate approach to determine the presence of functional gene (Straus et al., 1996).

Based on our analysis of the presence of virulenceassociated genes in the studied P. multocida strains we were unable to observe any association between the presence or absence of the tested virulence-associated genes and clinical representation of pasteurellosis in geese.

\subsection{Phylogenetic analysis}

Selected strains $(n=20)$ from the OMP groups were used to conduct thdF and rpoB-based phylogenetic analysis. The strains were selected based on their affiliation to the different OMP groups. The thdF and rpoB-based analysis revealed that seventeen strains were closely related phylogenetically to previously published strains of $P$. multocida subsp. multocida and P. multocida subsp. gallicida (Figs. 2 and S1), and only three strains from geese were grouped with previously published strains of $P$. multocida subsp. septica. By thdF and rpoBbased phylogenetic analysis, all these serotype A:1 arabinose-positive strains from the OMP group II were grouped closely to each other and to strains of $P$. multocida subsp. gallicida (Figs. 2 and S1). Due to their aberrant phenotype, we cannot say with certainty that these strains were $P$. multocida subsp. gallicida strains. However, arabinose-positive isolates among three subspecies of $P$. multocida were previously reported for $P$. multocida subsp. gallicida strains only (Kuhnert and Korczak, 2006; Mutters et al., 1985). Thus, we suspect that we have isolated atypical $P$. multocida subsp. gallicida strains from these particular geese.

\section{Discussion}

The results of our study indicate that $P$. multocida strains isolated from geese exhibit substantial phenotypic and genotypic diversity. This diversity could represent 


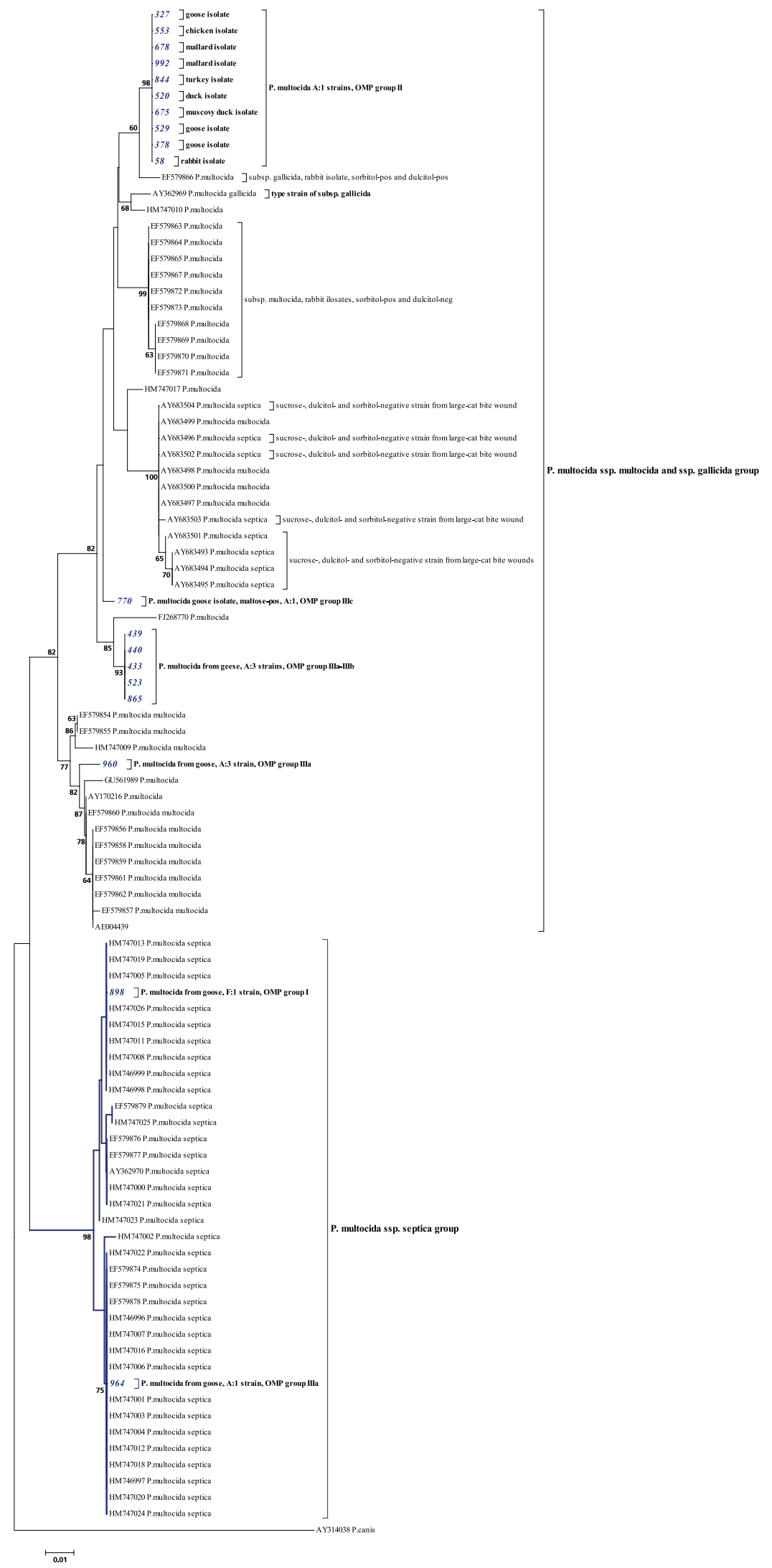


heterogeneity within the species isolated from infected geese. Previously, high levels of diversity have been demonstrated for avian, ovine and porcine isolates, in comparison to cattle respiratory isolates (Hotchkiss et al., 2011).

The OMP-based profiles of $P$. multocida geese strains revealed their clustering in three main groups. Group II was found to contain all the strains of $P$. multocida serotype $\mathrm{A}: 1$ that were isolated from peracute cases of $\mathrm{FC}$ in geese. All of the strains isolated from geese and assigned to group II expressed an unclassified biotype that was characterized by arabinose-positive and dulcitol- and xylose-negative reactions and by the weak ability of these strains to ferment sorbitol (Table S1). Dulcitol-negative P. multocida isolates that demonstrated weak and/or conflicting fermentation reactions in Andrade's sorbitol have been reported previously from infected dog and cat bite wounds (Hunt Gerardo et al., 2001), but similar strains have not been reported yet from poultry.

It has been reported that discrimination between subspecies of $P$. multocida, namely, $P$. multocida subsp. multocida, $P$. multocida subsp. septica, and $P$. multocida subsp. gallicida, can be ambiguous based on sorbitol, trehalose, and dulcitol fermentation reactions (Blackall et al., 1997; Christensen et al., 2004; Fegan et al., 1995; Hunt Gerardo et al., 2001).

Phylogenetically, the 16S rRNA gene sequences of strains of $P$. multocida subsp. multocida and strains of $P$. multocida subsp. gallicida were found to be almost identical, so use of the 16S rRNA gene was believed to be impracticable for the discrimination between these two subspecies. However, the $16 \mathrm{~S}$ rRNA gene sequences of strains of $P$. multocida subsp. septica, thought previously to form a phylogenetically separate group from the multocida/gallicida phylogenetic group, were later found to be a useful marker for the genetic identification of $P$. multocida subsp. septica (Petersen et al., 2001; Stahel et al., 2009). In addition, the other housekeeping genes, e.g. rpoB and $t h d F$, demonstrated higher variability than the 16S rRNA gene and were used as additional markers for phylogenetic studies and identification of Pasteurella isolates (Kuhnert and Korczak, 2006; Stahel et al., 2009). Based on previously published data about these genetic markers, we decided to use $r p o B$ and $t h d F$ genes for the assessment of phylogenetic grouping among our $P$. multocida strains as additional information to their observed biochemical characteristics.

The $t h d F$ and $r p o B$-based phylogenetic analysis of the 20 $P$. multocida strains selected from the three OMP groups revealed that 17 strains were closely related phylogenetically to previously published sequences for strains of $P$. multocida subsp. multocida and $P$. multocida subsp. gallicida; only three strains from geese were grouped with previously published sequences for strains of $P$. multocida subsp. septica.
In our study, the use of $t h d F$ and $r p o B$ gene sequencing allowed us to discriminate between the strains belonging to $P$. multocida subsp. septica group and the strains belonging to $P$. multocida subsp. multocida/gallicida group by comparison of sequence similarity. However, the ability of these markers to discriminate between the strains belonging to $P$. multocida subsp. multocida and $P$. multocida subsp. gallicida was not possible. From our experience, we can conclude that discrimination between $P$. multocida subsp. multocida and $P$. multocida subsp. gallicida strains based on the phylogenetic analysis of these housekeeping genes is problematic due to very limited information about the gene polymorphisms for P. multocida subsp. gallicida strains, which express the typical biochemical characteristics of this subspecies. Currently, only a few thdF and $r p o B$ gene sequences of $P$. multocida subsp. gallicida are available in GenBank.

In our study, two strains (P898 and P899) that belong phylogenetically to the $P$. multocida subsp. septica group expressed serotype $F: 1$ and were negative for the fermentation of dulcitol and trehalose but positive for the fermentation of xylose and sorbitol. Also, only these two strains were PCR-positive for the F serotype specific marker glycosyltransferase $(f c b D)$ gene (Townsend et al., 2001) and PCR-negative for putative filamentous hemagglutinin $(p f h A)$ and transferrin binding protein A $(t b p A)$ genes (Table S2). The absence of $p f h A$ and $t b p A$ genes in these two serotype $\mathrm{F}: 1$ strains could be explained by the main association of $p f h A$ with serogroups $B$ and $\mathrm{E}$, and $t b p A$ with serogroups $A$ and $B$, respectively. Another strain (P964) that belonged to $P$. multocida subsp. septica group based on the phylogenetic analysis of the thdF and $r p o B$ genes also expressed serotype A: 1 and was negative for the fermentation of dulcitol and positive for the fermentation of xylose, trehalose and sorbitol. The P964 strain was also found to be PCR-negative for the $t b p A$ gene but positive for the $p f h A$ gene. The presence of dulcitolnegative, sorbitol-positive strains of $P$. multocida subsp. septica has been previously reported (Hunt Gerardo et al., 2001). We agree with the authors of that study that sorbitol fermentation cannot be a reliable marker for differentiation among subspecies of $P$. multocida and additional phenotypic and/or genotypic tests are required for their unambiguous identification (Hunt Gerardo et al. 2001; Stahel et al., 2009).

In our phylogenetic analysis of $r p o B$ gene sequences of our strains compared with the gene sequences that are available in GenBank, we found eight previously published sequences for strains of $P$. multocida subsp. septica that were isolated from large-cat bite wounds and reported to be sucrose-, dulcitol- and sorbitol-negative strains (Christensen et al., 2005). These strains were not grouped with all other available sequences of $P$. multocida subsp. septica; however, they were grouped (with bootstrap supporting value of $100 \%$ ) with other

Fig. 2. Dendrogram showing phylogenetic relationships between $P$. multocida isolates from geese and $P$. multocida strains available in GenBank based on nucleotide sequence data for the $r p o B$ gene. The trees were constructed by the minimum evolution method in the MEGA 4 package. The bootstrap values presented at corresponding branches were evaluated from 1000 replications. GenBank accession numbers are indicated for each strain used in creating the dendrograms. 
P. multocida subsp. multocida (Fig. 2). Thus, this case may represent a taxonomic misidentification of sucrose-, dulcitol- and sorbitol-negative strains, which could represent atypical strains of $P$. multocida subsp. multocida. Further research is needed to find unambiguous markers for the differentiation between $P$. multocida subsp. multocida and P. multocida subsp. gallicida strains. Because the complete genome sequence of a common avian clone of P. multocida Pm70 is available now (May et al., 2001), we believe that genomic tools (i.e. microarrays, suppression subtractive hybridization and massive parallel sequencing) can be helpful for differentiation of these two subspecies and in the search for possible genetic markers (pathogenicity islands) of the $P$. multocida pathogenicity (Hert et al., 2008).

Finally, we can conclude that in our study the arabinose fermenting serotype A: 1 isolates of the $P$. multocida strains that expressed A type $p t f A$ gene were associated with peracute FC in the geese. The $P$. multocida strains of A:1 serotype have been previously reported to be associated with serious cases of fowl cholera (Christensen and Bisgaard, 2000; Leotta et al., 2006), including highly pathogenic "Vietnam type" strains of $P$. multocida that have been detected in Southeast Asia and also found to express serotype A:1 and the A type ptfA allele (Doughty et al., 2000; Wilkie et al., 2000). Further studies are required to examine the prevalence of these arabinose fermenting $P$. multocida strains in poultry in the European Union and other countries as well as the analysis of their contribution to causes of acute and peracute FC in geese.

\section{Acknowledgements}

We thank Drs. Maureen K. Davidson, Donna K. Chandler and Christine Anderson for their suggestions and critical review of this manuscript.

\section{Appendix A. Supplementary data}

Supplementary data associated with this article can be found, in the online version, at http://dx.doi.org/10.1016/ j.vetmic.2012.12.023.

\section{References}

Blackall, P.J., Pahoff, J.L., Bowles, R., 1997. Phenotypic characterisation of Pasteurella multocida isolates from Australian pigs. Vet. Microbiol. 57, 355-360.

Blanchong, J.A., Samuel, M.D., Mack, G., 2006. Multi-species patterns of avian cholera mortality in Nebraska's Rainwater Basin. J. Wildl. Dis. 42, 81-91.

Christensen, H., Angen, O., Olsen, J.E., Bisgaard, M., 2004. Revised description and classification of atypical isolates of Pasteurella multocida from bovine lungs based on genotypic characterization to include variants previously classified as biovar 2 of Pasteurella canis and Pasteurella avium. Microbiology 150, 1757-1767.

Christensen, H., Bisgaard, M., Angen, O., Frederiksen, W., Olsen, J.E., 2005. Characterization of sucrose-negative Pasteurella multocida variants, including isolates from large-cat bite wounds. J. Clin. Microbiol. 43, 259-270.

Christensen, J.P., Bisgaard, M., 2000. Fowl cholera. Rev. Sci. Tech. 19, 626637.

Davies, R.L., 2004. Genetic diversity among Pasteurella multocida strains of avian, bovine, ovine and porcine origin from England and Wales by comparative sequence analysis of the 16S rRNA gene. Microbiology 150, 4199-4210.

Davies, R.L., MacCorquodale, R., Baillie, S., Caffrey, B., 2003. Characterization and comparison of Pasteurella multocida strains associated with porcine pneumonia and atrophic rhinitis. J. Med. Microbiol. 52, 59-67.

Davies, R.L., Parton, R., Coote, J.G., Gibbs, H.A., Freer, J.H., 1992. Outermembrane protein and lipopolysaccharide variation in Pasteurella haemolytica serotype A1 under different growth conditions. J. Gen. Microbiol. 138, 909-922.

Doughty, S.W., Ruffolo, C.G., Adler, B., 2000. The type 4 fimbrial subunit gene of Pasteurella multocida. Vet. Microbiol. 72, 79-90.

Ewers, C., Lubke-Becker, A., Bethe, A., Kiebling, S., Filter, M., Wieler, L.H., 2006. Virulence genotype of Pasteurella multocida strains isolated from different hosts with various disease status. Vet. Microbiol. 114, 304-317.

Fegan, N., Blackall, P.J., Pahoff, J.L., 1995. Phenotypic characterisation of Pasteurella multocida isolates from Australian poultry. Vet. Microbiol. 47, 281-286.

Harper, M., Boyce, J.D., Adler, B., 2006. Pasteurella multocida pathogenesis: 125 years after Pasteur. FEMS Microbiol. Lett. 265, 1-10.

Hatfaludi, T., Al-Hasani, K., Boyce, J.D., Adler, B., 2010. Outer membrane proteins of Pasteurella multocida. Vet. Microbiol. 144, 1-17.

Heddleston, K.L., Gallagher, J.E., Rebers, P.A., 1972. Fowl cholera: gel diffusion precipitin test for serotyping Pasteurella multocida from avian species. Avian Dis. 16, 925-936.

Hert, D.G., Fredlake, C.P., Barron, A.E., 2008. Advantages and limitations of next-generation sequencing technologies: a comparison of electrophoresis and non-electrophoresis methods. Electrophoresis 29, 4618-4626.

Hotchkiss, E.J., Hodgson, J.C., Lainson, F.A., Zadoks, R.N., 2011. Multilocus sequence typing of a global collection of Pasteurella multocida isolates from cattle and other host species demonstrates niche association. BMC Microbiol. 11, 115.

Hunt Gerardo, S., Citron, D.M., Claros, M.C., Fernandez, H.T., Goldstein, E.J. 2001. Pasteurella multocida subsp. multocida and P. multocida subsp. septica differentiation by PCR fingerprinting and alpha-glucosidase activity. J. Clin. Microbiol. 39, 2558-2564.

Korbel, R., Gerlach, H., Bisgaard, M., Hafez, H.M., 1992. Further investigations on Pasteurella multocida infections in feral birds injured by cats. Zentralbl. Veterinarmed. B 39, 10-18.

Kuhnert, P., Korczak, B.M., 2006. Prediction of whole-genome DNA-DNA similarity, determination of $\mathrm{G}+\mathrm{C}$ content and phylogenetic analysis within the family Pasteurellaceae by multilocus sequence analysis (MLSA). Microbiology 152, 2537-2548.

Leotta, G.A., Chinen, I., Vigo, G.B., Pecoraro, M., Rivas, M., 2006. Outbreaks of avian cholera in Hope Bay, Antarctica. J. Wildl. Dis. 42, 259-270.

Lichtensteiger, C.A., Steenbergen, S.M., Lee, R.M., Polson, D.D., Vimr, E.R., 1996. Direct PCR analysis for toxigenic Pasteurella multocida. J. Clin. Microbiol. 34, 3035-3039.

May, B.J., Zhang, Q., Li, L.L., Paustian, M.L., Whittam, T.S., Kapur, V., 2001. Complete genomic sequence of Pasteurella multocida, Pm70. Proc. Natl. Acad. Sci. U.S.A. 98, 3460-3465.

Mutters, R., Ihm, P., Pohl, S., Frederiksen, W., Mannheim, W., 1985. Reclassification of the genus Pasteurella Trevisan 1887 on the basis of deoxyribonucleic acid homology, with proposals for the new species Pasteurella dagmatis, Pasteurella canis, Pasteurella stomatis, Pasteurella anatis, and Pasteurella langaa. Int. J. Syst. Bacteriol. 35, 309-322.

Petersen, K.D., Christensen, H., Bisgaard, M., Olsen, J.E., 2001. Genetic diversity of Pasteurella multocida fowl cholera isolates as demonstrated by ribotyping and 16S rRNA and partial atpD sequence comparisons. Microbiology 147, 2739-2748.

Petersen, K.D., Christensen, J.P., Bisgaard, M., 1998. Phenotypic and genotypic diversity of organisms previously classified as maltose positive Pasteurella multocida. Zentralbl. Bakteriol. 288, 1-12.

Samuel, M.D., Shadduck, D.J., Goldberg, D.R., Johnson, W.P., 2005. Avian cholera in waterfowl: the role of lesser snow and Ross's geese as disease carriers in the Playa Lakes Region. J. Wildl. Dis. 41, 48-57.

Sellyei, B., Banyai, K., Magyar, T., 2010. Characterization of the ptfA gene of avian Pasteurella multocida strains by allele-specific polymerase chain reaction. J. Vet. Diagn. Invest. 22, 607-610.

Stahel, A.B., Hoop, R.K., Kuhnert, P., Korczak, B.M., 2009. Phenotypic and genetic characterization of Pasteurella multocida and related isolates from rabbits in Switzerland. J. Vet. Diagn. Invest. 21, 793-802.

Straus, D.C., Jolley, W.L., Purdy, C.W., 1996. Characterization of neuraminidases produced by various serotypes of Pasteurella multocida. Infect. Immun. 64, 1446-1449. 
Tang, X., Zhao, Z., Hu, J., Wu, B., Cai, X., He, Q., Chen, H., 2009. Isolation, antimicrobial resistance, and virulence genes of Pasteurella multocida strains from swine in China. J. Clin. Microbiol. 47, 951-958.

Townsend, K.M., Boyce, J.D., Chung, J.Y., Frost, A.J., Adler, B., 2001. Genetic organization of Pasteurella multocida cap loci and development of a multiplex capsular PCR typing system. J. Clin. Microbiol. 39, 924-929.

Townsend, K.M., Frost, A.J., Lee, C.W., Papadimitriou, J.M., Dawkins, H.J., 1998. Development of PCR assays for species- and type-specific identification of Pasteurella multocida isolates. J. Clin. Microbiol. 36, 1096-1100.

Varga, Z., Sellyei, B., Magyar, T., 2007. Phenotypic and genotypic characterisation of Pasteurella multocida strains isolated from pigs in Hungary. Acta Vet. Hung. 55, 425-434.

Wilkie, I.W., Grimes, S.E., O'Boyle, D., Frost, A.J., 2000. The virulence and protective efficacy for chickens of Pasteurella multocida administered by different routes. Vet. Microbiol. 72, 57-68. 